\section{Cureus}

\title{
Hepatocellular Adenoma Presenting as a Focal Liver Lesion in Cirrhosis
}

Manoj K. Singh ${ }^{1}$, Michael R. Marvin ${ }^{2}$, David C. Wolf ${ }^{3}$, Manuel Rodriguez-Davalos ${ }^{4}$, Marcelo E. Facciuto ${ }^{5}$

1. Medanta Liver Institute, Medanta The Medicity 2. Division of Transplantation, Department of Surgery, University of Louisville, Louisville, KY 3. Liver Transplant Center, Westchester Medical Center 4. Department of Surgery, Yale University School of Medicine, New Haven, CT 5. Recanati Miller Transplantation Institute, Mount Sinai School of Medicine

$\square$ Corresponding author: Manoj K. Singh, msingh78@gmail.com

Disclosures can be found in Additional Information at the end of the article

\section{Abstract}

Hepatocellular adenoma is a benign neoplasm that almost always occurs in a histologically normal liver. We report the finding of a hepatocellular adenoma in the cirrhotic liver of a 56year-old female patient. A hypervascular nodule was detected by computed tomography (CT) scan and magnetic resonance imaging (MRI) conducted as part of a pre-transplant evaluation. The patient subsequently underwent orthotopic liver transplantation for a diagnosis of decompensated cirrhosis and suspected hepatocellular carcinoma. The pathological findings of the explant liver were consistent with adenoma in the setting of liver cirrhosis. We discuss the imaging findings and differential diagnosis, particularly in relation to a background of liver cirrhosis. We review the clinical situations when the diagnosis of adenoma can be supported, even in the setting of cirrhosis.

Categories: Internal Medicine, General Surgery, Oncology

Keywords: adenoma, cirrhosis, liver, nodule, imaging

\section{Introduction}

Hepatocellular adenoma is a benign neoplasm that almost always occurs in a histologically normal liver. We report the finding of a hepatocellular adenoma in the cirrhotic liver of a 56year-old female patient. A hypervascular nodule was detected by computed tomography (CT) scan and magnetic resonance imaging (MRI) conducted as part of a pretransplant evaluation. The patient subsequently underwent orthotopic liver transplantation for a diagnosis of decompensated cirrhosis and suspected hepatocellular carcinoma. The pathological findings of the explant liver were consistent with adenoma in the setting of liver cirrhosis. We discuss the imaging findings and differential diagnosis, particularly in relation to a background of liver cirrhosis. We review the clinical situations when the diagnosis of adenoma can be supported, even in the setting of cirrhosis.

Review began 01/19/2013

Published 01/31/2013

\section{Copyright 2013}

Singh et al. This is an open access article distributed under the terms of the Creative Commons Attribution License CC-BY 3.0., which permits unrestricted use, distribution, and reproduction in any medium, provided the original author and source are credited.

\section{Case Presentation}

A 56-year-old Caucasian female with a history of having been on birth control medication for 15 years in the past, and blood transfusion, had been diagnosed with chronic hepatitis $\mathrm{C}$. During routine follow-up, ultrasonography (US) of the abdomen did not show evidence of a liver mass, and a liver biopsy revealed chronic hepatitis with cirrhosis. Laboratory tests revealed transaminases in the $3 x$ normal range, with well-preserved hepatic function. The patient began a six-month course of therapy with alpha interferon but because of side-effects 


\section{Cureus}

and lack of improvement in liver chemistries, the medication was discontinued. The patient complained of severe fatigue and intermittent forgetfulness and was referred for liver transplant evaluation. CT scan of the abdomen demonstrated a nodular-appearing liver consistent with cirrhosis and a $2.5 \mathrm{~cm}$ solid lesion in the left lobe of the liver, with enhancement in the arterial phase. The serum alpha fetoprotein (AFP) level was $36.4 \mathrm{ng} / \mathrm{ml}$ for an upper limit of normal: $10.9 \mathrm{ng} / \mathrm{ml}$.

On subsequent MRI and US studies every three months, the size of the lesion remained stable. AFP levels ranged from $21.1 \mathrm{ng} / \mathrm{ml}$ to $28.2 \mathrm{ng} / \mathrm{ml}$. Appearance of the lesion on MRI was not conclusive for hepatocellular carcinoma (HCC), showing a contrast-enhanced lesion with faint hyperintensity on T1-weighted images and isointensity on T2-weighted images, and not clear washout on portal venous phase (Figures $1 A-1 C$ ).

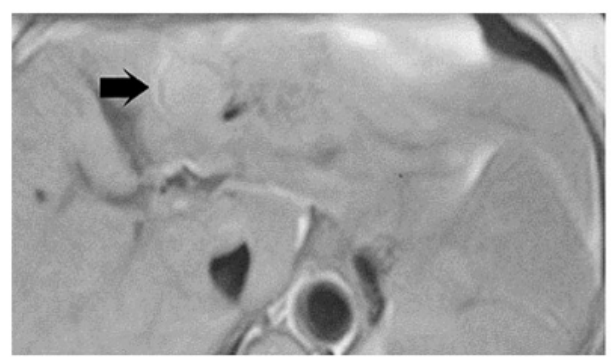

Figure 1A

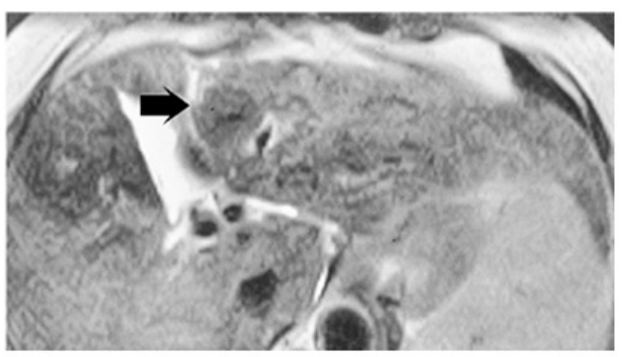

Figure 1B

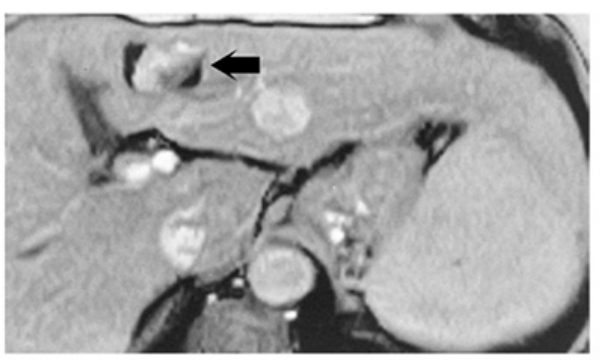

Figure 1C

\section{FIGURE 1: Appearance of the lesion on MRI was not conclusive for hepatocellular carcinoma (HCC)}

Figure 1A: T1-weighted MR image, the mass appeared to be surrounded by a capsule, with moderately higher signal intensity than liver parenchyma. Figure 1B: T2-weighted MR image shows the lesion to be isointense to the remainder of the liver. Figure 1C: MR image after infusion of contrast material shows an early blush of contrast enhancement.

Two attempts at CT-guided biopsy of the liver lesion revealed hepatitis C-induced cirrhosis, without evidence of malignancy. Metastatic work-up with bone scan and chest CT was negative.

One year after the lesion was first seen, US of the abdomen revealed a slight increase in the diameter of the solid hypoechoic lesion within the left lobe of the liver, to $3.5 \mathrm{~cm}$. Three months later, CT scan of the abdomen showed the mass had grown to $4.6 \mathrm{~cm}$ in diameter. The AFP level was $35.7 \mathrm{ng} / \mathrm{ml}$.

According to United Network for Organ Sharing (UNOS) policy for Liver Candidates with 


\section{Cureus}

Hepatocellular Carcinoma [1], the patient received extra priority in the waiting list for liver transplantation and she underwent orthotopic liver transplantation (OLT).

\section{Pathological Findings}

The liver was cirrhotic, with patent vasculature and biliary tree. The cut surface of the left lobe revealed a $3.5 \mathrm{~cm}$ well-circumscribed firm tan nodule (Figure 2).

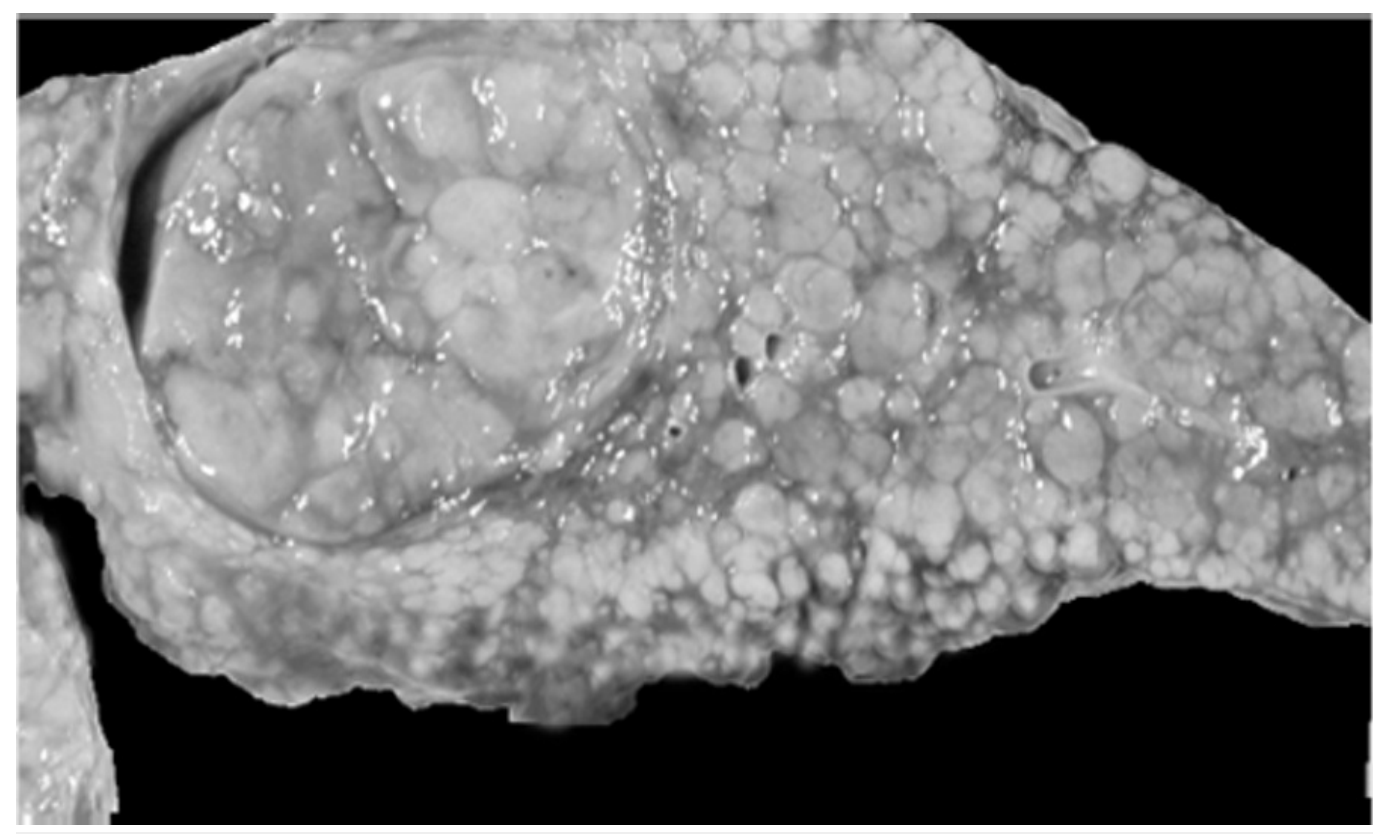

\section{FIGURE 2: A $3.5 \mathrm{~cm}$ tan firm nodule in the cut surface of the left lobe of the liver well demarcated from the surrounding cirrhotic parenchyma}

Microscopically, the nodule was composed of normal hepatocytes arranged in thick cords with marked vascularity. No portal tracts were noted in the entire nodule. The nodule margins were well-demarcated from the surrounding cirrhotic parenchyma. No necrosis, hemorrhage, or mitotic activity was seen. The pathological findings were compatible with the diagnosis of hepatocellular adenoma.

\section{Discussion}

Screening for HCC has become standard in the management of patients with cirrhosis or chronic hepatitis B/C infection. The most common screening tests are serum AFP and abdominal ultrasonography. AFP is not specific for HCC; it has a sensitivity of 39-64\%, specificity of $76-91 \%$ and a positive predictive value of $9-32 \%$. The addition of ultrasound scanning has increased the efficacy of surveillance. Ultrasonography has a sensitivity of $71 \%$ and specificity of $93 \%$, but its positive predictive value is $14 \%$ [2].

The detection of a focal lesion in a cirrhotic liver that is not clearly a hemangioma should raise the suspicion of HCC. The accuracy of HCC detection has improved with modern imaging techniques, such as contrast-enhanced CT and contrast-enhanced MRI, with reported sensitivities of $76 \%$ and $81.6 \%$, respectively [3-4]. However, radiological evaluation of a cirrhotic liver is complicated by the coexistence of regenerative nodules, dysplastic nodules, 
HCC, and benign tumors.

The sonographic appearance of HCC is variable. Small lesions tend to be hypoechoic. Larger lesions are generally hyperechoic. Some tumors have fibrous pseudocapsules. On spiral CT, a nodular lesion that enhances during the arterial phase and does not become hypointense in delayed phases is usually interpreted as a dysplastic nodule. On MRI, a nodule that is hyperintense on $\mathrm{T} 1$ - weighted images, isointense to hypointense on T2-weighted images, enhances during the arterial phase but does not become hypointense in delayed phases is generally considered a dysplastic nodule. HCC shows variable intensity on T1-weighted images, hyperintensity on $\mathrm{T} 2$-weighted images, enhancement during the arterial phase and washes out during delayed phases [5].

Dysplastic nodules are unusual nodules larger than the background regenerative nodules, with a different degree of dysplasia. Low-grade dysplastic nodules have a blood supply similar to that in the surrounding liver and are usually isodense on CT or MRI. High-grade dysplastic nodules have increased arterial blood supply and are commonly hyperintense on CT or MRI [6]. With increased size, there are more possibilities of malignancy; lesions greater than $20 \mathrm{~mm}$ are seldom benign [7].

Libbrecht, et al. reported a 2-8\% incidence of benign focal lesions in cirrhotic livers that were frequently interpreted as premalignant or malignant lesions on imaging before transplantation. Benign lesions detected in explant livers included focal nodular hyperplasia (FNH)-like nodules, infarcted regenerative nodules, foci of intrahepatic lithiasis, and bile duct adenomas. Several of these benign focal lesions enhanced on the arterial phase of contrastenhanced CT or MRI, a characteristic suggestive of HCC [8].

Pseudo-lesions due to arterioportal shunts often present as an arterial enhancing lesion in cirrhotic livers. They have a wedge form in subcapsular areas and do not cause bulging of the capsule [9].

Hepatocellular adenoma is defined as a benign neoplasm composed of hepatocytes. The lesion is hypervascular; serum AFP is normal or minimally elevated. The clinical setting is critical for the diagnosis of adenoma. Adenoma occurs in a setting of hepatocyte stimulation, usually with oral contraceptive, anabolic steroids, or abnormal carbohydrate metabolism as in familial diabetes mellitus, glycogen storage disease type 1a, and galactosemia. Malignant progression has been reported, but is rare. Adenoma almost always occurs within normal liver parenchyma; adenoma in a cirrhotic liver is rare, and this diagnosis should be made only if it can be supported by the presence of one of the accepted risk factors for adenoma [7]. In a series of 596 patients with cirrhosis on whom an autopsy was carried out, $0.7 \%$ of them were found to have hepatocellular adenoma [10].

In the present case, our patient had a known risk factor for adenoma with no history of hepatic mass from previous US. It is unclear whether the patient developed adenoma in the liver before becoming cirrhotic, and previous radiologic examination failed to demonstrate it, or the adenoma developed in a preexisting cirrhotic liver.

During pretransplant evaluation, a liver mass was first detected and slightly abnormal with no rising serum AFP. During radiological evaluation, the liver mass showed arterial enhancement on CT suggestive of HCC. However, the imaging characteristics of the lesion at MRI were consistent with a hepatic adenoma. Well-differentiated HCC can have similar imaging appearance, and in the setting of cirrhosis, this lesion was considered HCC until proven otherwise. A dysplastic nodule was considered in the differential diagnosis, although the finding of an arterial enhanced lesion more than $2 \mathrm{~cm}$ in size with a surrounding capsule made 
it unlikely. Given this unclear presentation of a presumed HCC, biopsy of the lesion was carried out, but because what we thought was a tissue sampling error, the definitive diagnosis was not achieved in the pretransplant course.

MRI during evaluation for liver transplantation in our patient correlated with the so-called gold standard pathological examination of complete explant liver. Adenomas usually are heterogeneous due to the presence of hemorrhage, necrosis or fat, and MRI is more definitive in detecting these specific features than CT [11].

The role of biopsy of hepatic lesions to confirm the diagnosis is controversial. Liver biopsy carries a $2 \%$ to $5 \%$ risk of tumor seeding and a $1 \%$ risk of bleeding. A negative biopsy of a nodule in a cirrhotic liver cannot be taken as a criterion to rule out malignancy. HCC in liver cirrhosis may be confidently diagnosed by imaging techniques if the lesion is greater than $2 \mathrm{~cm}$, hypervascular, of increased T2 signal intensity, hypointense in delayed phases (wash out), or associated with an elevated serum AFP level [12-13]. In less clear situations, a liver biopsy should be indicated if a positive result will prioritize the patient's transplantation status [14].

The detection of a hypervascular lesion in a cirrhotic liver has strong influence in patient management; it even provides extra priority to patients in the waiting list for liver transplantation [1]. In the clinical setting of cirrhosis, HCC is the main diagnosis to suspect, but it is also important to consider that benign conditions can simulate malignancy.

\section{Conclusions}

An awareness of this incidental finding of an adenoma in a cirrhotic liver, although uncommon, may contribute to the differential diagnosis of unclear focal lesions in cirrhotic livers in the setting of risk factors for adenoma.

\section{Additional Information}

\section{Disclosures}

Human subjects: All authors have confirmed that this study did not involve human participants or tissue. Conflicts of interest: In compliance with the ICMJE uniform disclosure form, all authors declare the following: Payment/services info: All authors have declared that no financial support was received from any organization for the submitted work. Financial relationships: All authors have declared that they have no financial relationships at present or within the previous three years with any organizations that might have an interest in the submitted work. Other relationships: All authors have declared that there are no other relationships or activities that could appear to have influenced the submitted work.

\section{References}

1. United Network for Organ Sharing. Policy 3.6. Allocation of Livers . (2002). Accessed: Feb, 2002: http://www.unos.org/.

2. Collier J, Sherman M: Screening for hepatocellular carcinoma. Hepatology . 1998, 27:273-278. 10.1002/hep.510270140

3. Lopez Hanninen E, Vogl TJ, Bechstein WO, Guckelberger O, Neuhaus P, Lobeck H, Felix R: Biphasic spiral computed tomography for detection of hepatocellular carcinoma before resection or orthotopic liver transplantation. Invest Radiol. 1998, 33:216-21.

4. Hirai K, Aoki Y, Majima Y, Abe H, Nakashima O, Kojiro M, Tanikawa K: Magnetic resonance imaging of small hepatocellular carcinoma. Am J Gastroenterol. 1991, 86:205-209.

5. Coakley FV, Schwartz LH: Imaging of Hepatocellular Carcinoma: A Practical Approach . Semin Oncol. 2001, 28:460-473. 10.1053/sonc.2001.26949

6. Hayashi M, Matsui O, Ueda K, Kawamori Y, Kadoya M, Yoshikawa J, Gabata T, Takashima T, 
Nonomura A, Nakanuma Y: Correlation between the blood supply and grade of malignancy of hepatocellular nodules associated with liver cirrhosis: evaluation by CT during intraarterial injection of contrast medium. Am J Roentgenol. 1999, 172:969-976.

10.2214/ajr.172.4.10587130

7. Anonymous: Terminology of Nodular Hepatocellular Lesions . Hepatology . 1995, 22:983-993.

8. Libbrecht L, Bielen D, Verslype C, Vanbeckevoort D, Pirenne J, Nevens F, Desmet V, Roskans T: Focal Lesions in Cirrhotic Explant Livers: Pathological Evaluation and Accuracy of Pretransplantation Imaging Examinations. Liver Transplantation. 2002, 8:749-761. 10.1053/jlts.2002.34922

9. Murakami T, Mochizuki K, Nakamura H: Imaging Evaluation of the Cirrhotic Liver . Semin Liver Dis. 2001, 21:213-224. 10.1055/s-2001-15497

10. Ruiz Guinaldo A, Martin Herrera L, Roldan Cuadra R: Hepatic tumors in patients with cirrhosis: an autopsy study. Rev Esp Enferm Dig. 1997, 89:771-780.

11. Federle MP, Brancatelli G: Imaging of Benign Hepatic Masses. Semin Liver Dis. 2001, 21:237249. 10.1055/s-2001-15344

12. Levy I, Greig PD, Gallinger S, Langer B, Sherman M: Resection of Hepatocellular Carcinoma Without Preoperative Tumor Biopsy. Ann Surg. 2001, 234:206-209. 10.1097/00000658200108000-00010

13. Bruix J, Sherman M, Llovet JM, Beaugrand M, Lencioni R, Burroughs AK, et al: Clinical Management of Hepatocellular Carcinoma. Conclusions of the Barcelona-2000 EASL Conference. J Hepatol. 2001, 35:421-430. 10.1016/S0168-8278(01)00130-1

14. Souto E, Gores GJ: When should a liver mass suspected of being a hepatocellular carcinoma be biopsied?. Liver Transpl. 2000, 6:73-75. 10.1002/lt.500060108 